\title{
Comparison between five typical reinforced honeycomb structures
}

\author{
Zhonggang WANG ${ }^{1,2, a^{*}, \text { Yiben ZHANG }}{ }^{1,2, b}$, Jiefu LIU ${ }^{1,2, c}$ \\ ${ }^{1}$ Key laboratory of Traffic Safety on Track, Ministry of Education, Changsha 410075 China; \\ ${ }^{2}$ School of Traffic \& transportation engineering, Central South University, Changsha 410075 China \\ awangzg_csu@163.com, ${ }^{\mathrm{b}}$ zhangyiben1228@126.com, ${ }^{\mathrm{c}}$ liujiefu1992@163.com
}

Keywords: honeycomb; reinforced structure; creative configuration; energy absorption.

Abstract. As cellular structure, high strength honeycomb is continuously pursued for last decades. This paper presents five typical reinforced honeycomb structures as new creative geometric configuration. Dynamic numerical simulations were conducted by means of finite element analysis. Corresponding dynamic response and mechanical properties were determined. In addition, comprehensive comparison between these five typical reinforced honeycomb structures was carried out in terms of mechanical behavior and energy absorption. The resultant verified that all these new five type reinforced structures perform perfect plateau stress, and the full-circle reinforced one has the best mechanical and energy absorption properties among all of them.

\section{Introduction}

Honeycomb structure has been a hot and preferential material in engineering application, due to its outstanding mechanical performance, particularly its specific energy absorption when loading at crash situation [1]. Hexagonal honeycomb structure is the most popular commercial product and has been widely used in kinds of industry fields, such as in aerospace, vehicles, high speed railway train and so on. Extensive research works were performed to determine its mechanical behavior with methodology [2] and experiments [3-4]. Some new types of energy absorption device were put forward [5]. In this investigation, five reinforced honeycomb structures were put forward, also, extensive numerical simulation were performed with the aim to determine the mechanical behavior and energy absorption of them. Section 2 detailed shows these typical reinforced honeycomb structure. Section 3 presents constructed full scale detailed finite element models. In addition, some discussion was carried out based on resultant response history curve and mechanical properties.

\section{Typical reinforced honeycomb structure}

Fig. 1 vividly shows five typical reinforced honeycomb structures. It can be seen that these five types of structures are both created based on the regular hexagonal cells. Basically, it can be categorized as three types. The first is simple reinforced with inside ribs. They are named as general and triangular type, just respectively as Fig. 1 (a) and Fig. 1 (b). The second is ribbed with another hexagon inside, they are respectively named as double hexagonal and full double hexagonal, just as Fig.1(c) and Fig.1 (d). Meanwhile the last type is reinforced with circle inside, and they are named as inside circular and full inside circular, just as Fig. 1 (e) and Fig. 1 (f). For general hexagonal honeycomb, it can be characterized with the thickness of cell $t$, the length of cell $h_{0}$. Whereas, for other four reinforced types, their length of inside hexagon or radius of inside circle are both half of $\mathrm{h}_{0}$, that means, $\mathrm{h}_{1}=\mathrm{h}_{0} / 2, \mathrm{R}=\mathrm{h}_{0} / 2$. Fig. 1 (g)

$\sim$ Fig. 1 (1) shows the detailed cells as below. 


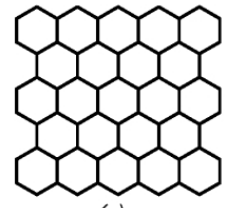

(a)

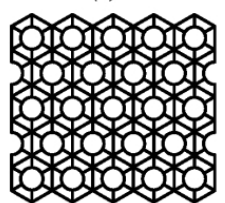

(d)

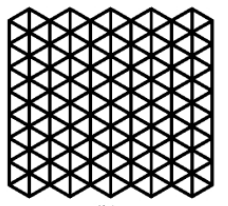

(b)

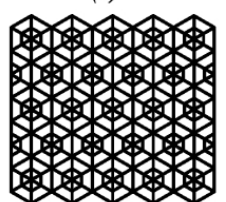

(e)

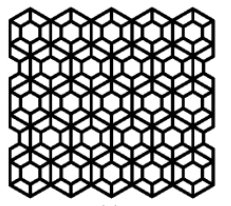

(c)

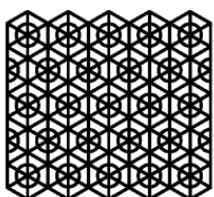

(f)

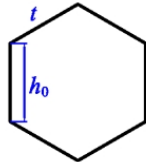

(g)

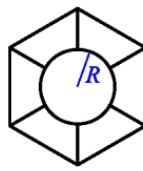

(j)

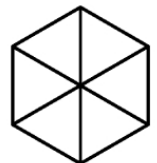

(h)

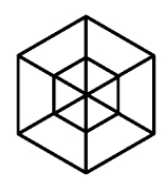

(k)

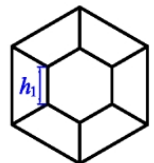

(i)

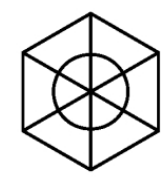

(l)

Fig.1 honeycomb structure with kinds of inside cells. (a) general structure; (b) Triangular;(c) double hexagonal; (d) inside circular; (e) full double hexagonal; (f) full inside circular; (g) cell of hexagonal; (h) cell of full-triangular; (i) cell of double hexagonal; (j) cell of inside circular; (k) cell of full inside hexagonal; (l) cell of full inside circular

\section{Simulation}

For each type of reinforced honeycomb structure, the finite element model was constructed with LS-DYNA $^{\mathrm{TM}}$ in APDL (ANSYS parametric dialogue language) [6]. Each model was composed of 13 Õ 11 cells, which completely satisfying the requirement according to Sun's convergence result presented in Ref. [7] that the mean out-of-plane dynamic plateau stress value becomes stable when cell number is no less than 8009 . The honeycomb core was $109 \mathrm{~mm}$ long, $110 \mathrm{~mm}$ wide and $60 \mathrm{~mm}$ thick in the overall dimension size.

The honeycomb cells were meshed by BT4 (Belytschko-Tsay-4node) shell elements and the mesh size was $1 \mathrm{~mm}$. The mechanical properties of foil material were as follows, density $2680 \mathrm{~kg} / \mathrm{m}^{3}$, Young's module $69.3 \mathrm{GPa}$, Poisson ratio 0.33 , yield stress $215 \mathrm{MPa}$. In this investigation, the foil material was treated as an ideal elastic-plastic constitution model. Automatic single surface contact algorithm was applied to avoid penetration between cell walls. The specimen was placed on a static rigid plate as a boundary and crushed by another moving rigid plate at a constant velocity as presented in Ref. [8].

Fig. 2 presents the numerical simulation results of these five typical reinforced honeycomb structures together with the regular one. Fig.2 (a) presents all of the dynamic plane stress versus compression ratio vividly, and Fig.2 (b) shows their energy absorption against compression ratio. For convince of comparison, the plane stresses as well as energy absorption per volume are calculated. Table 1 reported the corresponding mean stress $\sigma_{m}$, specific mean stress $\sigma_{s}$, energy absorption per volume $E_{v}$, specific energy absorption $E_{s}$ (by weight). One should be pointed out that $\sigma_{m}$ is the average plane stress of the plateau stage, the specific mean stress was calculated as $\sigma_{s}=\sigma_{m} / \rho_{s}$, where $\rho_{s}$ is the equivalent density of the numerical model. At the same time, the energy absorption is the integral of plane stress of the whole response history against the compression before densification. The specific energy absorption $E_{s}$ was calculated with the mass of numerical finite element models.
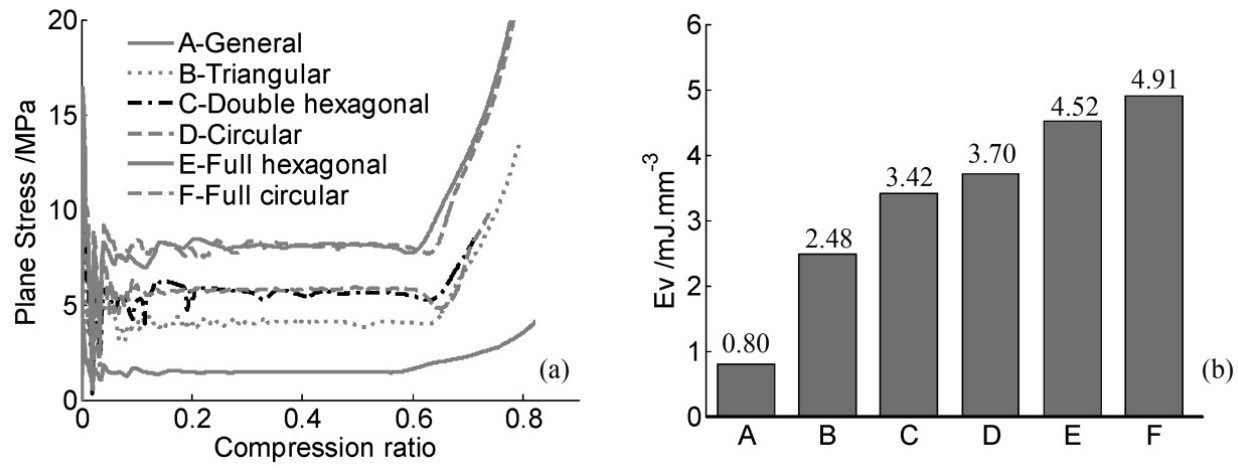

Fig.2 Numerical simulation results. (a) response curves; (b) energy absorption 
Table 1 Simulation results $(\mathrm{t}=0.06 \mathrm{~mm}, \mathrm{~h}=4 \mathrm{~mm}, \mathrm{l}=4 \mathrm{~mm})$

\begin{tabular}{lcccc}
\hline \multicolumn{1}{c}{ Type } & $\begin{array}{c}\sigma_{m} \\
{[\mathrm{MPa}]}\end{array}$ & $\begin{array}{c}\sigma_{s} \\
{\left[\mathrm{kN} \cdot \mathrm{m}_{\mathrm{kg}}{ }^{-1}\right]}\end{array}$ & $\begin{array}{c}E_{v} \\
{\left[\mathrm{~mJ} . \mathrm{mm}^{-3}\right]}\end{array}$ & $\begin{array}{c}E_{s} \\
{\left[\mathrm{~kJ} . \mathrm{kg}^{-1}\right]}\end{array}$ \\
\hline A-General & 1.48 & 30.88 & 0.80 & 16.62 \\
B-Triangular & 4.07 & 29.12 & 2.48 & 17.73 \\
C-Double hexagonal & 5.61 & 40.25 & 3.42 & 24.56 \\
D-Circular & 5.83 & 41.23 & 3.70 & 26.22 \\
E-Full hexagonal & 8.09 & 43.76 & 4.52 & 24.42 \\
F-Full circular & 8.17 & 43.70 & 4.91 & 26.27 \\
\hline
\end{tabular}

From the Fig. 2 and Table 1, it can be clearly seen that the geometric configuration has a significant influence on mechanical behaviors, both on mean stress and energy absorption. From the original pure simple hexagonal honeycomb structure (A-General) to the last full reinforced circular (F-Full circular), the mean stress jumps from 1.48 $\mathrm{MPa}$ to $8.17 \mathrm{MPa}$. And the energy absorption per volume steps up to $4.91 \mathrm{~mJ} . \mathrm{mm}^{-3}$ from $0.80 \mathrm{~mJ} . \mathrm{mm}^{-3}$. This huge promotion is evident and significant, which is closely related to the density of reinforced honeycomb structure. After all, more cell walls contribute higher energy absorption.

Further comparison between the specific mean stress and specific energy absorption were carried out. Fig. 3 shows the detailed bar graph as below.
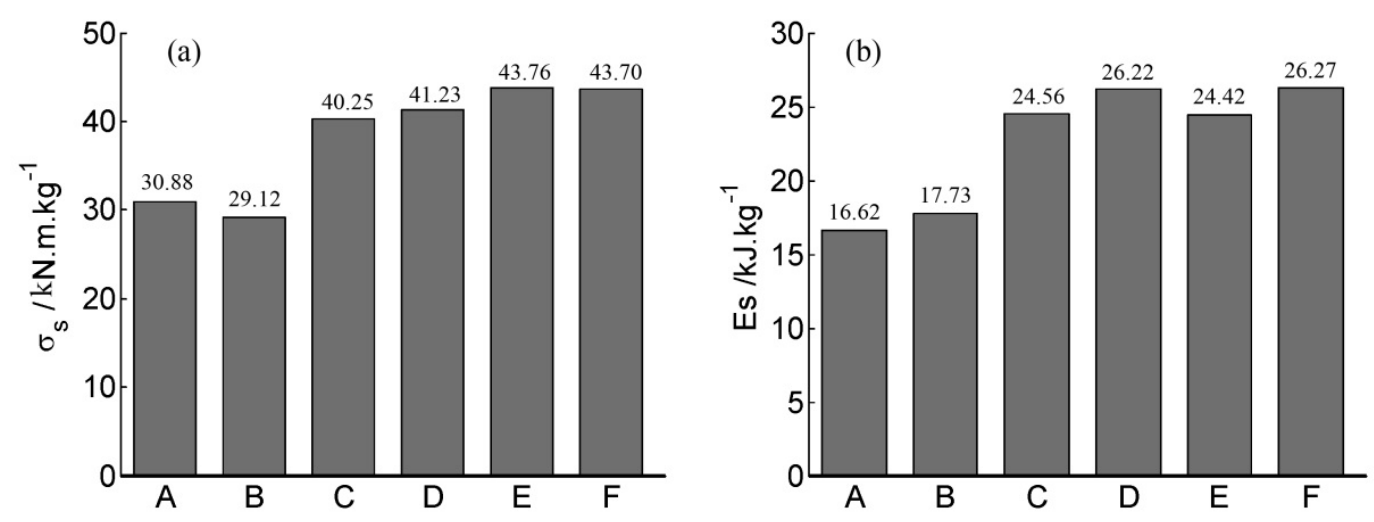

Fig.3 Comparison of mechanical properties. (a) Specific mean stress; (b) Specific energy absorption

From the bar and figures, it can be found that the ribbed patter with circular performs better than other types. At the same time, the creative structure in geometric configuration reinforced with inside hexagon or circle shows outstanding specific mean stress as well as the specific energy absorption. They nearly keep on the same level, but obviously higher than the general and triangular structures.

\section{Conclusions}

Based on the analyses mentioned above, it can be concluded that all these five reinforced honeycomb structure contribute great improvement not only in the load-carrying capacity but also in energy absorption ability. Among all these five typical reinforced structure, the ribbed patter with inside circle is better than triangular and hexagonal type. This reinforced way is recommend.

\section{Acknowledgements}

This work was financially supported by The National Natural Science Foundation of China (51505502), and The Key Union Project of National Natural Science Foundation of China on High-Speed Railway (U1334208). This support is acknowledged with thanks. 


\section{References}

[1] Gibson LJ, Ashby MF. Cellular solids, structures and properties. 2nd ed. Cambridge, UK:

Cambridge University Press, 1997.

[2] Wang Z, Tian H, Lu Z, et al. Mechanics of Advanced Materials and Structures, online, 2015.

[3] Wang Z, Tian H, Lu Z, et al. Composites Part B: Engineering, 2014, 56: 1-8.

[4] Wang Z, Lu Z. Journal of Central South University (Science and Technology), 2013; 44(3):1246-1251. In Chinese.

[5] ZhonggangWang,GuangjunGao, HongqiTian,et al. International Journal of Crashworthiness, 2013, $18(5), 483-491$.

[6] Hallquist J. LS-DYNA user's manual, 970ed. Livemore Software Technology Corporation,2003.

[7] Sun DQ, Zhang WH and Wei YB. Compos Struct 2010;92 (11):2609-2621.

[8] Wang Zhong-gang,LU Zhai-jun,XiaXi., Journal of Materials Engineering,2013, (5):78-82,88. In Chinese. 